ISSN 1112-9867

http://www.jfas.info

\title{
EVALUATING THE MOVEMENT OF ACTIVE FAULTS ON BURIED PIPELINES
}

\author{
Y. Parish \\ Assistant Professor and PhD in Civil Engineering - Research and \\ Educational Complex in Azerbaijan (Ministry of Energy)
}

Published online: 15 May 2016

\begin{abstract}
During the earthquake, a buried pipeline may be experienced extreme loading that is the result of the relatively large displacement of the Earth along the pipe. Large movements of ground could occur by faulting, liquefaction, lateral spreading, landslides, and slope failures. Since the pipelines are widely spread, and in some areas they pass necessarily the fault areas so it is important to study improvement of pipelines in areas with faults. In this paper, it has been preceded to the active movement of the fault on buried pipelines.
\end{abstract}

Keywords: Water Installation, Earthquakes, Vibrations, Faults, Pipe

Author Correspondence, e-mail: yousefparish@yahoo.com

doi: http://dx.doi.org/10.4314/jfas.v8i3s.245

\section{INTRODUCTION}

Large deformations caused by the failure of slopes, earthquakes, faults move and float tubes in shallow trenches has created major injuries in the network of buried pipelines. Because of extend buried pipelines in urban communities such as Tehran that in fact these lines have formed entangled skein the various factors causing damage to the pipelines should be considered in the design of buried pipelines. Due to the longevity of underground pipelines and exploitation period in environmental conditions and also bylaws changes, the necessity of 
upgrading, retrofitting, preventative, reparation is critical against destructive agents. One of the destructive phenomena on the pipelines is the movement of fault. In recent years, many researchers have studied on the effect of the movement of active faults on buried pipelines. Obviously, the usable of pipelines after the movement of fault needs to be able inelastic deformation without making damage.

The earthquake may cause serious damage to water installation of a city. Several statistics and reports exist from around the world about gaining extreme damages caused by accidents on Lifelines of various installations such as water, gas, electricity and telecommunications after the occurrence of an earthquake with high intensity.

Such reports are available since the 1906 earthquake in San Francisco, America so far. In this earthquake the water purgation installation and pipelines of the city had damaged extremely. In a separate report, the matter of major earthquakes Mexico City in September 1985 has been propounded. In this earthquake that has led to the displacement range of land, meantime the destruction of tanks and drinking water treatment plant, main pipelines of drinking water also broke and as the result of that more than 4 million people didn't have drinking water for three weeks.

Also the installation of preparation drinking water and Main transmission and distribution of water pipelines had broken due to the permanent destruction of the ground in 1994 Northridge earthquake in California.

\section{EFFECTS OF DIVERSE GROUND MOVEMENT ON BURIED PIPELINES}

New gas pipelines, whether coastal or marine are usually buried to provide the reliance protection of them. Buried pipelines are designed typically based on limitations belt tension for internal pressure. For pipelines exposed to extreme temperature changes may be required especial tension analyses in terms of bending. In addition pipelines may need to design for external loads, for example, the loads due to heavy equipment on the ground. A buried pipeline may be experience severe loading during the earthquake, that is the result of the relative large displacement of the ground along the pipe. Large movements of the ground may be created by faulting, liquefaction, lateral spreading, landslides, and slope failures. However, 
it can't be avoided from the movements due to faulting and liquefaction like lateral spreading over long distances of pipelines in areas with high seismicity.

Ground interruption patterns related to movements caused by earthquakes are usually complex and show significant changes in displacement as a function of geological formations, soil type and magnitude and duration of earthquakes.

The pipeline operation in the areas with constant movements of ground closely depends on the applying or non-applying of compressive or tensile deformation on the pipeline. A pipeline will be affected by bending and axial deformation in the occasion that large fault displacements exist.

Two modes of deformation are possible in the situations that a buried pipeline be strongly compressed. The Pipeline may be isolated from the soil like an arrow be buckled upward or may be involved local distortion and shrinkage of the septum in shell.

Although the pipeline is relatively shapeable in stretch, but the focus of Tanjesh may be resulted due to weaknesses of welding, changes in thickness of wall, heterogeneity of yielding point and .... It is recommended that to be considered the preparation for significantly limiting the maximum tensile Tanjesh in amount of less than the total capacity of the pipeline materials Tanjesh.

\section{GEOTECHNICAL INVESTIGATIONS}

Faulting is the main factor and cause to arise the earthquake. Faults are long extensions that land layers and classes have been broken along these stretches and cause to produce a band or the Zone. This failure often are steep and not vertically. Thus, a surface is mounted on the other surface in terms of the weight. Place and extension of these faults can be identified according to the region tectonic maps.

Earthquakes occur as a result of movements of geodynamic Earth and its surfaces. By earthquakes a surface goes to beneath another surface and resulting energy from this movement release as seismic waves, due to the linearity of some underground Structures, some of these structures may encounter with faults and even in some cases is inevitable. Since the displacement of the fault is very malicious and structure design is not affordable in a way 
that be strength in the face of these tensions, most forcefully be recommended to consider to places and extension of faults in designing and implementation of long underground structures (tunnels, subways and ...) and to be avoided intersections of Structures with extension of this faults as much as possible.

On the other hand, the study of fault movements and displacement can provide valuable information for engineers that can be determined amount of fault moves (vertical and horizontal). Fault displacement varies with increasing depth and even these movements is not the same along the fault

Determination the amount of this displacement and Practical underground structure is important in designing the structure and decision-making in the face of faults this means that a movement no matter how small will have destructive effect on some of the structures and in opposite such movements is less important on some other structures with different using such channels.

Pipelines should be placed in the way that to be away from ground discontinuities or be placed and protected in the way to minimize deformation due to lasting Movements of ground.

A single building or group of buildings are often enclosed in a small area and in such cases geotechnical program is designed to provide an accurate picture of the subsurface conditions. In contrast, transmission lines and distribution networks may cover a wide area so that the focused study on the entire length of the line is not possible.

Since the performance of detailed geotechnical studies is not possible in the whole path of a pipeline, it is important to ensure from adaptation of existence conditions with design assumptions in drilling pipeline.

The designer must consider the Specific information that is obtained in geotechnical studies to proper designing of pipelines for resistance against lasting deformations of the ground at the intersection of faults.

This information include: the geometry of the fault (s), the extent of the fault zone, the type, amount and direction of movement that occurs during the earthquake suddenly or gradually over the long term (creep), and Recurrence time interval for a given amount of displacement. 
Perhaps the most important factor that should be considered in seismic design of pipelines at the intersection of active faults is the amount and type of surface displacement. The amount of displacement of the fault's surface is perfectly linked with the size of the earthquake (magnitude and length of the fault rupture). Other factors seem to have effect on the amount of surface displacement such as the type of fault movement (strike-slip, normal, etc.) and ordering tectonic but available data is not enough to determine their importance.

\section{EFFECTIVE FACTORS ON THE PERFORMANCE OF THE PIPELINE AT THE INTERSECTION OF THE FAULT}

Factors that substantially are involved in the pipeline operation at exposed the fault movements are: the depth of burial, the form of the moat, the amount of relative displacement of fault, the angle of intersection of the pipeline with the fault, soil properties and effective uninhibited lengths. The operation of pipes is also affected by material properties, geometry of the pipeline and its internal pressure. Generally, the dynamic answer of pipeline is small in comparison with the deformation caused by fault and can be ignored so that good results can be obtained from a static operated Relative changing location.

Other factors that have effect on performance of pipeline include pipe diameter and wall thickness, internal pressure and material properties of pipes. Among these, the wall thickness is the main factor at the intersections leading to the tension. Longitudinal forces acting on the pipeline from the surrounding soil are proportional to the pipe diameter, yet the resistance of pipeline against these forces is proportional to the product of the diameter and thickness.

The ratio of longitudinal force on the resistance of the pipeline determines the effective long inhibitory of the pipe line. So this length is directly proportional to the thickness of the pipe wall. As a result, capability of the fault intersection is independent from the pipe diameter and is directly with pipe wall thickness. Increasing the pipeline wall will lead to reduction in Tanjesh tension at the faults intersection whereas the other factors will not be changed.

There is some possibility for pipelines which are subject to lateral pressure of ground to being oval ring, unless a limit is applied above the ratio of pipeline diameter to wall thickness. 
Internal pressure not only reduces being oval ring but also improves resistance of pipelines against Local shrinkage by reducing the effects of unstable initial geometric defect.

\section{BURIED PIPELINES IN DEALING WITH FAULTS}

Buried pipelines systems are damaged at the time of earthquake and this damage has caused a lot of problems in everyday life. Since, the pipelines are widely spread, and in some areas they pass the fault areas necessarily. So studying the improvement of pipelines has special importance in areas with faults. Loading caused by the earthquake is applied to the pipe by fault displacement. As a result of that, this displacement causes to creating force and tension in the buried pipelines.

Fracture of buried pipelines is as the fracture caused by the interaction of axial force and bending torsion. The sensitivity of design parameters must be evaluated at the fracture of the pipelines. The results show that increasing the angle of friction between soil and tube become closer to their fracture region with increasing shift fault, increasing the pipe diameter, increasing the depth of buried pipes.

However, early studies showed that whatever the fault movement is larger, the length of deformed part of the pipe will be longer. So the changeable form zone should be considered larger and shift amount should be considered greater if you use flexible joints or rails along the lines.

The solutions that are suggested for further understanding of the critical points include:

- Ultrasonic tests for thickness measurement pipe walls have been used to evaluate the effects of corrosion of pipes that have been placed in depth of the ground, places with difficult access, height, adjacent of the ceilings or inside the dropped ceilings.

- To evaluate the Columns and foundations, bracing frames, pipes and bracing steel Structures include investigating and testing the safety and quality of welds through non-destructive testing (NDT) and testing Ultrasonic testing (UT), liquid penetrant testing (MT), magnetic particle testing.

- radiography and radiographic (RT) check device status and fluid transfer equipment that placed along with pipelines, such as pumps, compressors and... 
- It can be mentioned changing plan, change profile, structural measures, systematic measures, changing plan in accordance with the amount of the faults movement as measures to cope with earthquake in the crossing pipelines with faults. These measures are divided into two categories: hardware measures and software measures, described as follows:

- Hardware measures: hardware measures have been formed from structural measures and measures based on changing the plan. By adopting this strategy, it is possible to preserving the operation of the pipeline even after the earthquake; the fault displacement is covered by these measures in small to medium-scale.

- Software measures: software Measures has been formed from changing basis plan and systematic measure. The fault displacement in medium to large-scale, that they aren't applicable with above-mentioned hardware measures, is covered by these measures. In this measures cannot be avoided from the extent of the damage and the aim of these measures is to maintain performance or minimize the loss of performance.

- Reviewing used materials.

- Checking the connection, splits and their resistance.

\section{ANALYZING THE PIPELINES UNDER THE INFLUENCE OF FAULT MOVEMENT}

The most common method to tolerance for welded steel pipelines is the maximum using from capacity of fault deformation in the range of inelastic in stretch in order to coordinate with ground deformation without rupture. Whenever it is possible, the placing of the pipeline in crossing with the fault line must be chosen in the way that the pipeline under the influence of the stretch has been placed with some bending. It must be avoided to placing the pipe line in the way that it becomes impacted because the capacity of the pipeline in tolerance of pressured tanjesh is much less than stretching tanjesh. 
Several methods exist for analyzing the buried pipelines in exposed to fault. These methods with needful reforming in terms of constraints can be used for buried pipelines and same cases. These methods include:
1) Newmark-Hall Method
2) Kennedy and colleagues Method
3) Finite Element Method

The first two methods assume the fault movement in the way that occurs in a defined single page and consider the soil aggregates on both sides of fault to two rigid motion bodies. Newmark-Hall method provides a lower bound estimate of tanjesh in the pipeline. Because it assumes that the pipeline can be separated from the ground that as a result deletes any side constraint. The pipeline will be deformed to a straight line between two inhibitory points. On the other hand Kennedy and colleagues method leads to the upper boundary estimation of tanjesh.

The displacement by fault movement is naturally three-dimensional and depends on the components of extension Slip and normal slip of the fault. So movements of anchorage points of the pipeline depend on the manner of pipeline arrangement than the upper surface of the fault and deflection of fault surface than the upper surface.

A pipeline system is usually spread over a large area, and this feature, put the system at additional risk compared to other facilities that occupy small area. It is when faulting and considering to main parameters that effect on the issue.

These parameters include:
A) Surrendering the pipe under axial and bending load by forming plastic hinges and axial sliding
B) Longitudinal friction at the interface between pipe and soil.
C) lateral resistance of soil

Seismic analyzing of buried pipelines Exposed to fault is an important task that includes a complex interaction of soil issue - structure with numerically numerous problems, such as:

1) Three-dimensional geometry

2) Large deformations 
3) Local buckling cross

4) Eulerian buckling under compression fault

5) The pipe slip Due to the around soil

6) Non-linear behavior of the soil

Designing pipelines for the transit of fault

In designing pipelines for transit of the fault, the following considerations usually improve pipeline capacity for resistance against heterogeneous fault movements:

- Where practicable, the angle of intersection of the pipeline than to strike-slip fault must be chosen such that the pipeline to be placed under tension.

- Depth of buried pipelines in fault zones to be minimal as much as possible to reduce frictional resistance. If there is no threat on surface, it is the better to place the pipes on the ground.

- Increasing the wall thickness of the pipe will raise the capacity of the pipeline to fault movement in a given level. If the Relatively thick pipe is applied on both sides of the fault.

- Precise control of embankments around the pipe is necessary within close distances to the fault. Generally loose granular to semi-dense soil or without large hunts or Rubble is suitable for embankment.

- Placing the pipeline on built Levee with low frictional resistance

- Placing the pipeline at larger trenches than necessary and filling them with resistant and appropriate materials.

- Smooth and covering surface be used as much as possible to reduce friction, such as fiberglass or epoxy that should be flat but hard.

- At The fault location it is better to the pipeline to be direct and be away from the curvature burial depth is not to be changed and the pipeline doesn't have no elbows, tees, flanges and similar constraints in the near-fault as much as possible. 


\section{DISCUSSION AND CONCLUSION}

Although the pipeline is relatively formable in stretch, but it is possible to Tanjesh focus due to the weaknesses of welding, the changing of the walls thickness, heterogeneity yield point and etc. is resulted. For this reason, it is recommended that to be considered measures to limit the maximum tensile Tanjesh in significant amounts less than the ultimate capacity of Tanjesh of the pipeline materials. Since the displacement of the fault is very destructive and designing the structure is not affordable so that be resistant in the face of these tensions It is recommended most forcefully to consider to location and Extension of faults in designing and implementation of underground long Structures (tunnels, subways and...) and to be avoided crossing the structures with extension of these faults as far as possible.

At the other side the study of fault displacement and movements can provide valuable information that could help engineers to determine the amount of fault movement (vertical and horizontal).

Fault displacements increases with depth, and even these movements are not the same along the fault. The pipelines must be placed in the way to be away from ground failures or be placed and protected to deformation of the steady movements of the ground become minimal. The amount of the fault surface displacement is completely associated with the size of the earthquake (means magnitude and length of the fault rupture). Dynamic response of the pipeline as compared to the deformation caused by ruptured is small and can be ignored So that good results can be obtained from an applied relative lateral static change.

The capability of fault intersection is independent to pipeline diameter and directly with wall thickness of the pipe. Increasing pipeline wall will lead to reduction in tension Tanjesh at the intersection of faults. It provided that all other factors remain unchanged.

It is suggested to be considered following points in designing the structures in the event that preventing dealing the structures with fault is not possible:

-Use flexible connectors in the place of dealing the structures with the fault

- Use intentional loopholes to centralize the damages caused by earthquake

- Increasing cross-section the size of the expected shift from earthquake 
The most common method for welded steel pipelines to tolerate the fault displacement is maximum using from deformation capacity of the pipeline in the range of inelastic in the stretch in order to coordination with ground deformation without rupture.

\section{REFERENCES}

Dabaghi, M, plumbing retrofit installations, in 1385, the first International Conference on Seismic Retrofit

Rajai, $\mathrm{H}$, evaluate the behavior of the fault lines, the first International Conference on Seismic Retrofit

Komakpanah, Ali, Montazerghaem, Saeid, International Institute of Seismology and Earthquake Engineering, Proceedings of the First Workshop on examining strategies for reducing the damages of the earthquake in Iran, Tehran, 1994.

Earthquake damage and countermeasure of industrial lifeline andequipment, Yonglu Li \& Xiangdong Xi.

Earthquakes and Seismic Faulting: Effects on Tunnels, VILLY A. KONTOGIANNI \& STATHIS C. STIROS.

Joe Nane (joe) Wang., June 1993.

Kiezo Ohtomo, Toshio Shiero, Tadashi Kawai, Kensye Kanaya, Experimental aspect of laminar shear sand box excitation test whit embedded RC model.

Seismic behavior of underground structures and site response, Y.M.A.Hashash

How to cite this article:

Parish Y. Evaluating the movement of active faults on buried pipelines. J. Fundam. Appl. Sci., 2016, 8(3S), 1126-1136. 